\title{
Erratum to: An Asthma and Diabetes Quality Improvement Project: Enhancing Care in Clinics and Community Health Centers
}

\author{
Alan Silver • James Figge • Donna L. Haskin • \\ Veronica Pryor - Karen Fuller • Thomas Lemme • \\ Nancy Li • Mary Jane O'Brien
}

Published online: 13 February 2011

(C) Springer Science+Business Media, LLC 2011

Erratum to: J Community Health

DOI 10.1007/s10900-010-9296-8

A revised version of Table 3 is presented here to correct errors in the originally published version.

The online version of the original article can be found under doi:10.1007/s10900-010-9296-8.

\footnotetext{
A. Silver $(\bowtie) \cdot$ V. Pryor · T. Lemme $\cdot$ N. Li IPRO Health Care Quality Improvement Team, 1979 Marcus Ave, Lake Success, NY 11042, USA

e-mail: asilver@ipro.org

J. Figge · D. L. Haskin · K. Fuller · M. J. O’Brien New York State Department of Health Office of Health Insurance Programs Team, Albany, NY, USA
} 
Table 3 Diabetes-ages 18-64

\begin{tabular}{|c|c|c|c|c|c|}
\hline \multirow[t]{2}{*}{ Indicator } & \multicolumn{2}{|l|}{ Baseline } & \multicolumn{2}{|l|}{ Follow-up } & \multirow[t]{2}{*}{$P$ value } \\
\hline & Numerator/denominator & $\%$ & Numerator/denominator & $\%$ & \\
\hline Completed cases & 1,091 & & 404 & & \\
\hline Female & $729 / 1,091$ & 66.82 & $244 / 404$ & 60.40 & 0.3956 \\
\hline Black/Hispanic & $716 / 1,091$ & 65.63 & $254 / 404$ & 62.87 & 0.0148 \\
\hline Had 4 or more clinic visits during the sample period & $793 / 1,091$ & 72.69 & $278 / 404$ & 68.81 & 0.0149 \\
\hline Documentation of comorbidity & $937 / 1,091$ & 85.88 & $397 / 404$ & 98.27 & $<0.0001 *$ \\
\hline Most recent blood pressure $\leq 140 / 90$ & $749 / 1,089$ & 68.78 & $316 / 403$ & 78.41 & 0.3264 \\
\hline Most recent blood pressure $\leq 130 / 80$ & $488 / 1,089$ & 44.81 & $232 / 403$ & 57.57 & 0.0302 \\
\hline $\mathrm{HbA1c}$ tested at least once & $945 / 1,088$ & 86.86 & $376 / 400$ & 94.00 & $<0.0001 *$ \\
\hline HbA1c tested at least twice & $547 / 1,088$ & 50.28 & $227 / 388$ & 58.51 & $0.0004 *$ \\
\hline HbA1c value $\geq 9$ & $292 / 945$ & 30.90 & $86 / 376$ & 22.87 & 0.0322 \\
\hline HbA1c between $8-8.9$ & $169 / 945$ & 17.88 & $52 / 376$ & 13.83 & 0.1167 \\
\hline HbA1c between 7-7.9 & $184 / 945$ & 19.47 & $77 / 376$ & 20.48 & 0.1533 \\
\hline $\mathrm{HbA} 1 \mathrm{c}<7$ & $300 / 945$ & 31.75 & $158 / 364$ & 43.41 & 0.0540 \\
\hline Lipid profile performed at least once & $636 / 1,089$ & 58.40 & $340 / 401$ & 84.79 & $<0.0001 *$ \\
\hline $\mathrm{LDL}>130 \mathrm{mg} / \mathrm{dl}$ & $210 / 631$ & 33.28 & $61 / 339$ & 17.99 & $<0.0001 *$ \\
\hline LDL between $101-130 \mathrm{mg} / \mathrm{dl}$ & $186 / 631$ & 29.48 & $75 / 339$ & 22.12 & 0.0236 \\
\hline $\mathrm{LDL} \leq 100 \mathrm{mg} / \mathrm{dl}$ & $218 / 631$ & 34.55 & $184 / 339$ & 54.28 & $<0.0001 *$ \\
\hline Triglycerides $<150$ & $308 / 631$ & 48.81 & $193 / 339$ & 56.93 & 0.1610 \\
\hline $\mathrm{HDL}>40$ & $434 / 631$ & 68.78 & $199 / 339$ & 58.70 & 0.1237 \\
\hline Urine Microalbumin performed at least once & $421 / 1,091$ & 38.59 & $221 / 404$ & 54.70 & $<0.0001 *$ \\
\hline Foot examination performed and documented & $525 / 1,085$ & 48.39 & $270 / 403$ & 67.00 & $<0.0001 *$ \\
\hline Dilated retinal exam performed and documented & $112 / 1,085$ & 10.32 & $118 / 403$ & 29.28 & $<0.0001 *$ \\
\hline Dilated retinal exam referral but no report on record & $430 / 973$ & 44.19 & $185 / 285$ & 64.91 & $<0.0001 *$ \\
\hline On ACEI or ARB & $602 / 813$ & 74.05 & $304 / 337$ & 90.21 & $<0.0001 *$ \\
\hline On aspirin/antiplatelet therapy & $374 / 1,018$ & 36.74 & $263 / 393$ & 66.92 & $<0.0001 *$ \\
\hline Flu vaccination & $534 / 1,028$ & 51.95 & $230 / 374$ & 61.50 & $0.0006^{*}$ \\
\hline Documentation of diabetes patient education & $616 / 1,091$ & 56.46 & $311 / 404$ & 76.98 & $<0.0001 *$ \\
\hline Smoking cessation discussed & $95 / 231$ & 41.13 & $94 / 121$ & 77.69 & $<0.0001 *$ \\
\hline
\end{tabular}

All models control for differences between clinics and types of Medicaid coverage

* Significant after the Bonferroni correction; with 27 measurements in the analysis, the significant level is $0.05 / 27=0.0019$ 